\title{
Anatomia dos ramos do nervo lingual em suíno
}

\author{
Anatomy of branches of nerve lingual in pigs \\ Jussara Rocha Ferreira ${ }^{{ }^{*}}$ Patrícia Marques Fortes ${ }^{\text {II }}$ Alessandra Guerra Dias ${ }^{\text {III }}$ \\ Paulo Roberto de Sousa ${ }^{I}$
}

\section{RESUMO}

Neste trabalho, foram estudadas as ramificações e a distribuição do nervo lingual em 18 línguas de suínos adultos de ambos os sexos, sem especificação de raça. $O$ material, colhido a fresco em rotina de abate, foi resfriado $\left(4^{\circ} \mathrm{C}\right)$ para transporte, fixado em solução aquosa de formaldeído (7\%, 72 horas), imerso em solução aquosa de ácido nítrico (15\%, 72 horas), e dissecado sob lupa (RANSOR - II-20). Cada antímero dissecado possibilitou o registro dos ramos nervosos linguais primários, secundários e terciários presentes no interior das estruturas miofasciais do órgão, exibindo diferentes arranjos: o antímero esquerdo apresentou quatro a 10 ramos primários, quatro a 12 ramos secundários e zero a 11 ramos terciários. Já o antímero direito apresentou três a 8 ramos primários, zero a 11 ramos secundários e zero a 10 ramos terciários. Em 27,6\% das análises, os ramos linguais estabelecem associações com as fibras do nervo hipoglosso no antímero correspondente.

Palavras-chave: língua, suínos, nervo lingual, nervo craniano, sistema nervoso.

\section{ABSTRACT}

The distribution and ramifications of the lingual nerve of 18 tongues of adult pigs from both genders with no strain specification were studied. The material freshly collected in slaughter routine was cooled $\left(4^{\circ} \mathrm{C}\right)$ for transportation, fixed in formaldehyde aqueous solution $(7 \%, 72 \mathrm{~h})$, immerse in nitric acid aqueous solution $(15 \%, 72 h)$ and dissected with magnifying glass (RASOR, II-20). Each dissected antimere enabled the recording of primary, secondary and tertiary lingual nervous branches present inside the myofascial structure of the organ, exhibiting different arrangements: left antimere: 4 to 10 primary branches, 4 to 12 secondary antimeres and 0 to 11 tertiary branches; right antimere: 3 to 8 primary branches, 0 to 11 secondary antimeres and 0 to 10 tertiary branches. In $27.6 \%$ of analyses, the lingual branches established associations with the fibers of the hypoglossal nerve in the corresponding antimere.

Key words: tongue, pigs, lingual nerve, cranial nerve, nervous system.

\section{INTRODUÇÃO}

Os suínos, animais historicamente domesticados pelas populações humanas, representam uma importante espécie do sistema produtivo na cadeia dos agronegócios. Domesticar um animal para produção significa fazer constantes ajustes no desenho corporal desta espécie para atender demandas de mercado. A conversão alimentar é fundamental para garantir saúde, aleitamento e ganho de peso, sendo que estas questões estão relacionadas à palatabilidade do alimento, que é resultante da inervação sensitiva e motora da língua.

Na literatura publicada na área, percebe-se que alguns autores se referem aos nervos glossofaríngeo e lingual como os grandes responsáveis pela motricidade e pela percepção sensorial da sofisticada mucosa lingual em ungulados (SONNTAG, 1922; BRUNI e ZIMMERL, 1951; SHAWARZE \& SCHRODER, 1972; GETTY, 1981), em carnívoros

\footnotetext{
Instituto de Ciências Básicas, Universidade Federal de Goiás (UFG). *Endereço para correspondência: Faculdade de Medicina, Universidade Federal de Brasília (UnB), Campus Universitário Darcy Ribeiro, Área de Morfologia, Laboratório de Pesquisa em Macro e Mesoscopia, Sala BC-321, Asa Norte, 70910-900, Brasília, DF, Brasil. E-mail: jussararocha@unb.br.

IIPrograma de Ciências da Saúde, Faculdade de Medicina, UFG, Goiânia, GO, Brasil.

IIIDepartamento de Morfologia, ICB/UFG, Gioiânia, GO, Brasil.
} 
(OKANO, 1953) e em primatas (CASTELLI e HUELKE, 1964; VIG e KANAGASUNTHERAN, 1969; FAVAROLO, 1979; BITTENCOURT et al., 1987; MADEIRA, 2004). Também é considerável o número de estudos a respeito da microinervação dispersa entre o tecido conjuntivo lingual na zona muscular ou na papilar (SCALZI, 1967; MIKHAIL et al., 1980; SEMPRINI et al., 1985).

Com base nestas observações, optou-se por estudar os ramos linguais dependentes do nervo lingual em suínos e suas subdivisões macro e mesoscópicas. O objetivo é contribuir para o conhecimento morfológico do sistema digestório a partir das hipóteses de GETTY (1981), que considera que plexos linguais motores representam fibras dos nervos hipoglosso e lingual distribuídas entre os músculos da língua de ungulados, e de SONNTAG (1920 e 1925), que considera a complexidade do desenho da língua uma adaptação evolutiva, na busca das espécies pela disputa por alimentos em seus diferentes habitats. Isso inclui a diversidade morfológica da membrana mucosa.

\section{MATERIAL E MÉTODOS}

Foram dissecados 36 antímeros linguais de suínos, de ambos os sexos, cujas idades variaram entre seis e 12 meses para fins de estudo dos ramos linguais dependentes do nervo lingual. Em vinte e sete antímeros, houve sucesso na sistematização dos ramos linguais do nervo em três hierarquias de calibre. O material, colhido a fresco em rotina de abate (Frigorífico Goiás Carne, na região metropolitana de Goiânia-Goiás), foi resfriado $\left(4^{\circ} \mathrm{C}\right)$ para transporte e, em seguida, fixado em solução aquosa de formaldeído (7\%) por 72 horas, imerso em solução aquosa de acido nítrico (10\%) por 72 horas. A dissecação ocorreu sob lupa, dissecandose a musculatura intrínseca e extrínseca por meio da superfície ventral da língua para visualização dos ramos nervosos (RANSOR, II-20). Para fins de quantificação e esquematização linear, a sistematização dos ramos do nervo lingual obedeceu aos critérios estabelecidos por BITTENCOURT et al. (1987), inclusive para a denominação dos ramos do nervo cuja terminologia adotada foi a seguinte: ramos linguais primários, secundários e terciários do nervo lingual. Esta nomenclatura não está contemplada na NÔMINA ANATÔMICA VETERINÁRIA(1994).

\section{RESULTADOS E DISCUSSÃO}

Em 36 antímeros de língua de suínos, foram estudados os ramos linguais dependentes do nervo lingual destinados à mucosa, à musculatura da língua e outros tecidos componentes do órgão. O nervo lingual representa um ramo calibroso do nervo mandibular. Sua origem aparente ocorre medialmente ao côndilo mandibular, em tronco comum com o nervo alveolar inferior, em ungulados, conforme ilustrou SCHALLER(1999).

De trajetória caudorostral descendente, o tronco principal do nervo lingual percorreu o espaço pterigomandibular medialmente ao corpo da mandíbula e às fibras da porção interna do ventre do músculo masseter. Após percorrer um trajeto látero medial, o tronco principal do nervo lingual alcançou o espaço ventral existente na base da língua, abaixo da mucosa do soalho da boca, lateralmente à linha de reflexão desta mesma mucosa em relação ao freio lingual. A mucosa a partir do freio lingual em sentido caudal delimita abaixo desta um espaço em cada antímero ocupado pelo músculo milohióideo, que representa um diafragma relativamente completo. Lateralmente, os espaços subjacentes são ocupados pelas glândulas sublinguais e ducto das glândulas submandibulares, bilaterais. Entre e acima destas glândulas e do músculo genioglosso, as fibras do nervo lingual alcançam o assoalho da cavidade oral distribuindo-se na mucosa do soalho da boca, nas pregas franjadas e na prega sublingual para a seguir se projetar no corpo lingual.

Na região que antecedeu a inserção do freio lingual, os ramos nervosos alcançaram medialmente o corpo da língua. A partir do ponto de penetração no parênquima lingual até atingir o ápice lingual, foi freqüente a associação (Figura 1) de fibras do nervo lingual com fibras do nervo hipoglosso. Ao abordar o corpo da língua, os ramos linguais do nervo lingual adotaram uma seqüência de subdivisões cuja topografia nos permitiu classificá-las em subdivisões primárias, secundárias e terciárias. Isso foi observado em 27 dos 36 antímeros analisados (Tabelas 1 e 2 e Figuras 2, 3 e 4).

Esta análise se ateve à descrição macro e mesoscópica dos ramos linguais em suas subdivisões aqui interpretadas como primárias, secundárias e terciárias, conforme proposição de BITTENCOURT et al. (1987), ao estudar este nervo em humanos, sendo que esta interpretação não é equivalente em outros vertebrados. Vale destacar que SHALLER (1999) representou em esquemas alguns ramos dos nervos linguais, mas o autor não nomina suas subdivisões.

A dissecação possibilitou sistematizar os ramos linguais do nervo lingual nos dois terços rostrais da língua, visto que os feixes nervosos alcançaram o órgão, penetrando neste no sentido dorso ventral entre os músculos intrínsecos, onde se subdividiram a partir de ramos denominados primários (Tabela 1 e Figura 2). Em outras duas ordens de grandeza, foram vistos os 


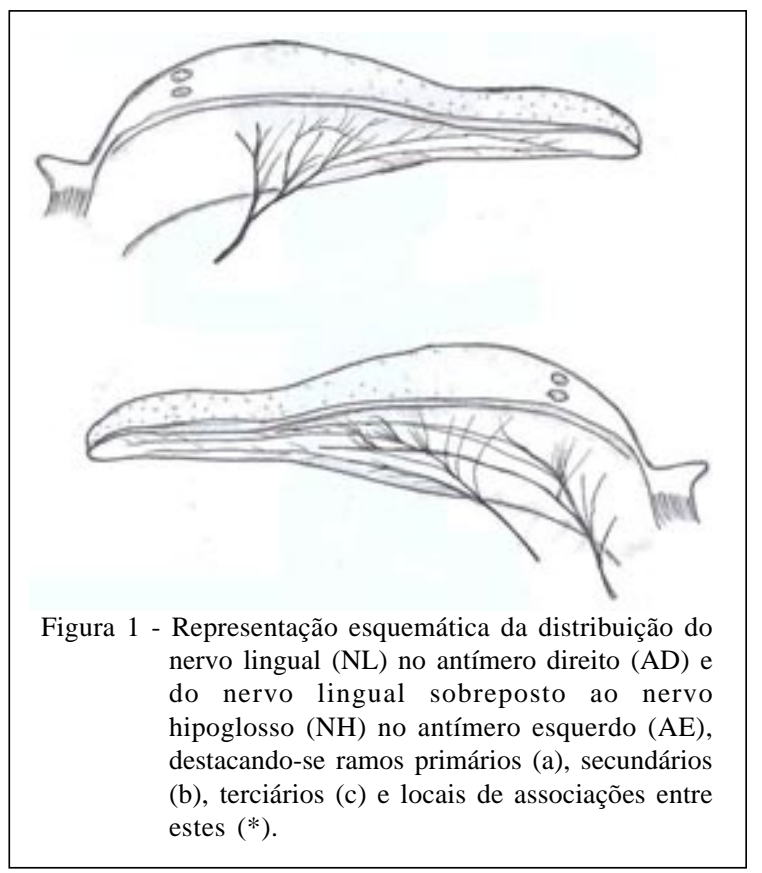

ramos secundários (Tabela 1 e Figura 3), cujas presenças dependeram de um ramo precedente, sendo estes em geral mais curtos e restritos a uma região específica. Os ramos terciários foram interpretados como subdivisões finais, porém observáveis macroscopicamente (Tabela 1 e Figura 4). Neste nível foi freqüente a presença de pequenos plexos em sítios pontuais do parênquima do órgão (esquema na Figura 4).

A distribuição espacial da subdivisão do nervo nos antímeros, no interior da musculatura e seus envoltórios conjuntivos, aconteceram como segue: no antímero direito foram vistos entre três e oito ramos primários, zero e onze ramos secundários, zero e dez ramos terciários; no antímero esquerdo, nas 27 oportunidades, foram observados de quatro a 10 ramos primários, quatro a 12 ramos secundários e de zero a 11 ramos terciários (Tabela 1 e Figuras 2, 3 e 4).

Em 11 antímeros (37,93\%), foram observadas associações entre os ramos secundários e terciários do nervo lingual com fibras do nervo hipoglosso (Figura 1), possibilidade pelos autores em ungulados e carnívoros (GETTY, 1989; DYCE et al., 1990), além de humanos (MADEIRA, 2004; BITTENCOURT et al. 1987).

Entre os autores, DYCE et al. (1990) consideraram que, embora os músculos intrínsecos sejam supridos pelo nervo hipoglosso, entendido como motor da língua, é provável que fibras sensoriais que emanam de feixes e de outros receptores nos músculos sigam principalmente pelo nervo lingual. A literatura sinalizou também que o nervo lingual conduz fibras aferentes gustatórias provenientes de papilas linguais (MADEIRA, 2004; GETTY, 1981; SCALZI, 1967; SEMPRIMI et al., 1985) da região do ápice e cdo orpo lingual, além de fibras eferentes viscerais parassimpáticas (MADEIRA, 2004).

$\mathrm{Na}$ verdade, pode-se entender que as chamadas ligações ou intercomunicações referidas pelos autores em nível plexioforme submucoso, subepitelial ou intramuscular (GETTY 1981; WATANABE \& KONIG, 1977; MIKHAIL et al., 1980; SEMPRINI et al., 1985) representem não intercomunicações propriamente ditas, mas sim associações de fibras nervosas, cujas origens (seja na mucosa, seja nas miofáscias) foram áreas onde os nervos se sobrepõem em territórios comuns ou de inervação múltipla. A análise macro e mesoscópica demonstrou, em nível de plexos terciários, a riqueza de sobreposição de inervação na zona próxima à mucosa. Naturalmente que estas observações não puderam ser quantificadas, em razão de sua pequena ordem de grandeza e da limitação metodológica desta análise.

Conforme este estudo demonstrou, as subdivisões macroscópicas primárias dos ramos linguais do nervo lingual ocorreram em $100 \%$ das oportunidades observadas, o que vai ao encontro do relato dos autores em ungulados (GETTY, 1981 e SCHALLER, 1999) e em cães (DYCE et al., 1990; EVANS e CHRISTENSEN, 1979). As divisões secundárias encontradas em $96,2 \%$ dos antímeros analisados e as terciárias em 88,8\% dos casos estudados não tiveram descrições equivalentes em animais domésticos. Em humanos, FAVAROLO (1979) relatou a existência de 12 a 15 ramos de nervos distribuídos na língua e BITTENCOURT et al. (1987) descreveram com clareza estas subdivisões, sendo que seus estudos em humanos foram usados como proposta metodológica para análise no suíno. Os resultados foram, dentro da similaridade possível, ao encontro do estudo destes autores. Outro trabalho (BLANDIN, 1938) reportou a presença de inúmeros ramos nervosos dirigidos à mucosa lingual sem, no entanto, sistematizar critérios de quantificação.

SONNTAG (1920 e 1925), ao tratar da descrição comparativa, classificação e filogenia da língua em diversas ordens animais, destacou que os ungulados têm duas partes distintas na língua, incluindo um ápice livre e tátil e uma parte posterior com função muscular mecânica. O mesmo autor destacou também que naquelas línguas em que se desenvolveram papilas valadas e órgãos laterais (as papilas folhadas), ou seja, papilas mecânicas anteriormente e papilas gustativas posteriormente, aconteceu um avanço evolutivo na sofisticação 
Tabela 1 - Quantificações dos ramos linguais primários, secundários e terciários do nervo lingual por indivíduo e por antímero, e as associações com o nervo hipoglosso em Sus scrofa doméstica (L. 1758). Goiânia, 2008.

\begin{tabular}{|c|c|c|c|c|c|}
\hline \multirow[b]{2}{*}{ Número do Caso } & \multirow[b]{2}{*}{ Antímero AE/AD } & \multicolumn{3}{|c|}{------------------Número de ramos------------------ } & \multirow[b]{2}{*}{ Presença de associações } \\
\hline & & ramos primários & ramos secundários & ramos terciários & \\
\hline \multirow[t]{2}{*}{01} & $\mathrm{AE}$ & 4 & 4 & - & - \\
\hline & $\mathrm{AD}$ & 3 & 3 & 2 & + \\
\hline \multirow[t]{2}{*}{02} & $\mathrm{AE}$ & 9 & 5 & 4 & - \\
\hline & $\mathrm{AD}$ & $\ldots$ & $\ldots$ & $\ldots$ & $\ldots$ \\
\hline \multirow[t]{2}{*}{03} & $\mathrm{AE}$ & 10 & 12 & 8 & - \\
\hline & $\mathrm{AD}$ & 8 & 8 & 1 & - \\
\hline \multirow[t]{2}{*}{04} & $\mathrm{AE}$ & 7 & 8 & 4 & + \\
\hline & $\mathrm{AD}$ & 5 & 4 & 4 & - \\
\hline \multirow[t]{2}{*}{05} & $\mathrm{AE}$ & 6 & 11 & 11 & - \\
\hline & $\mathrm{AD}$ & $\ldots$ & $\ldots$ & $\ldots$ & $\ldots$ \\
\hline \multirow[t]{2}{*}{06} & $\mathrm{AE}$ & 6 & 4 & 5 & + \\
\hline & $\mathrm{AD}$ & $\ldots$ & $\ldots$ & $\ldots$ & $\ldots$ \\
\hline \multirow[t]{2}{*}{07} & $\mathrm{AE}$ & 6 & 5 & 2 & - \\
\hline & $\mathrm{AD}$ & 5 & 2 & - & - \\
\hline \multirow[t]{2}{*}{08} & $\mathrm{AE}$ & $\ldots$ & $\ldots$ & $\ldots$ & $\ldots$ \\
\hline & $\mathrm{AD}$ & 7 & 6 & 2 & - \\
\hline \multirow[t]{2}{*}{09} & $\mathrm{AE}$ & 6 & 4 & 2 & + \\
\hline & $\mathrm{AD}$ & 8 & 4 & 5 & - \\
\hline \multirow[t]{2}{*}{10} & $\mathrm{AE}$ & $\ldots$ & $\ldots$ & $\ldots$ & $\ldots$ \\
\hline & $\mathrm{AD}$ & 8 & 11 & 7 & + \\
\hline \multirow[t]{2}{*}{11} & $\mathrm{AE}$ & $\ldots$ & $\ldots$ & $\ldots$ & $\ldots$ \\
\hline & $\mathrm{AD}$ & 7 & 9 & 4 & - \\
\hline \multirow[t]{2}{*}{12} & $\mathrm{AE}$ & $\ldots$ & $\ldots$ & $\ldots$ & $\ldots$ \\
\hline & $\mathrm{AD}$ & 5 & 10 & 4 & + \\
\hline \multirow[t]{2}{*}{13} & $\mathrm{AE}$ & 8 & 4 & 3 & + \\
\hline & $\mathrm{AD}$ & 6 & 4 & 3 & - \\
\hline \multirow[t]{2}{*}{14} & $\mathrm{AE}$ & 6 & 5 & 2 & - \\
\hline & $\mathrm{AD}$ & 8 & 10 & 10 & + \\
\hline \multirow[t]{2}{*}{15} & $\mathrm{AE}$ & 7 & 7 & 1 & - \\
\hline & $\mathrm{AD}$ & 6 & 6 & 2 & + \\
\hline \multirow[t]{2}{*}{16} & $\mathrm{AE}$ & 9 & 9 & 4 & + \\
\hline & $\mathrm{AD}$ & 6 & 7 & 6 & - \\
\hline \multirow[t]{2}{*}{17} & $\mathrm{AE}$ & $\ldots$ & $\ldots$ & $\ldots$ & $\ldots$ \\
\hline & $\mathrm{AD}$ & 8 & - & - & + \\
\hline \multirow[t]{2}{*}{18} & $\mathrm{AE}$ & 7 & 5 & 7 & - \\
\hline & $\mathrm{AD}$ & $\ldots$ & $\ldots$ & $\ldots$ & $\ldots$ \\
\hline
\end{tabular}

Fonte: Dados de Pesquisa UFG/UnB. Ferreira et al., 2008.

Notas: sinal convencional utilizado: (...) dado não disponível; (-) dado numérico igual a zero; (AE) antímero esquerdo; (AD) antímero direito; (+) associação presente.

vásculo-nervosa. O suíno se enquadra nesta interpretação, ou seja, tanto a musculatura quanto a superfície mucosa se especializaram a ponto de haver, seja na base ou no corpo e ápice lingual, sobreposição de território de inervação entre os ramos dos nervos linguais e hipoglosso (Tabela 1 e Figura 1).

Pode-se concluir que os ramos do nervo lingual formam plexos distribuídos amplamente na musculatura intrínseca da língua e na mucosa subjacente; os ramos linguais primários do nervo lingual são formações constantes (100\% das observações); os ramos linguais do nervo lingual distribuem-se nas miofascias da língua tendo sido denominados de ramos primários, secundários e terciários, de acordo com a hierarquia de subdivisões; os ramos primários não participaram da inervação do parênquima lingual; secundários e terciários do nervo lingual se distribuíram por entre os componentes

Ciência Rural, v.38, n.9, dez, 2008. 
Tabela 2 - Freqüências absolutas e relativas do total de ramos primários, secundários e terciários do nervo lingual em línguas de suínos (Sus scrofa domestica, L. 1758). Goiânia, GO, 2008.

\begin{tabular}{|c|c|c|c|c|}
\hline \multicolumn{3}{|c|}{-------Números de ramos------- } & \multirow{2}{*}{$\begin{array}{c}\text { freqüência } \\
\text { absoluta }\end{array}$} & \multirow{2}{*}{$\begin{array}{l}\text { freqüência } \\
\text { relativa }\end{array}$} \\
\hline primários & secundários & terciários & & \\
\hline 03 & & & 01 & $3,70 \%$ \\
\hline 04 & & & 01 & $3,70 \%$ \\
\hline 05 & & & 03 & $11,11 \%$ \\
\hline 06 & & & 08 & $29,62 \%$ \\
\hline 07 & & & 05 & $18,51 \%$ \\
\hline 08 & & & 06 & $22,22 \%$ \\
\hline 09 & & & 02 & $7,40 \%$ \\
\hline 10 & & & 01 & $3,70 \%$ \\
\hline & 0 & & 01 & $3,70 \%$ \\
\hline & 02 & & 01 & $3,70 \%$ \\
\hline & 03 & & 01 & $3,70 \%$ \\
\hline & 04 & & 07 & $25,92 \%$ \\
\hline & 05 & & 04 & $14,81 \%$ \\
\hline & 06 & & 02 & $7,40 \%$ \\
\hline & 07 & & 02 & $7,40 \%$ \\
\hline & 08 & & 02 & $7,40 \%$ \\
\hline & 09 & & 02 & $7,40 \%$ \\
\hline & 10 & & 02 & $7,40 \%$ \\
\hline & 11 & & 02 & $7,40 \%$ \\
\hline & 12 & & 01 & $3,70 \%$ \\
\hline & & 0 & 03 & $11,11 \%$ \\
\hline & & 01 & 02 & $7,40 \%$ \\
\hline & & 02 & 06 & $22,22 \%$ \\
\hline & & 03 & 02 & $7,40 \%$ \\
\hline & & 04 & 06 & $22,22 \%$ \\
\hline & & 05 & 02 & $7,40 \%$ \\
\hline & & 06 & 01 & $3,70 \%$ \\
\hline & & 07 & 02 & $7,40 \%$ \\
\hline & & 08 & 01 & $3,70 \%$ \\
\hline & & 10 & 01 & $3,70 \%$ \\
\hline & & 11 & 01 & $3,70 \%$ \\
\hline Total: 52 & Total: 77 & Total: 57 & Total: 79 & \\
\hline
\end{tabular}

Fonte: Dados de Pesquisa UFG/UnB. Ferreira et al., 2008.

Notas: sinal convencional utilizado: (...) dado não disponível; (-) dado numérico igual a zero; (AE) antímero esquerdo; (AD) antímero direito.

miofaciais e outros tecidos formando plexos; em nove dos 27 antímeros analisados verificou-se a existência de associações entre ramos dos nervos lingual e hipoglosso, indicando nestes sítios uma superposição de inervação; a inervação da língua de suínos se enquadra, na interpretação de SONNTAG (1925), como as demais línguas de ungulados que adquiriram complexidade morfológica, estabilidade e maturidade evolutiva.

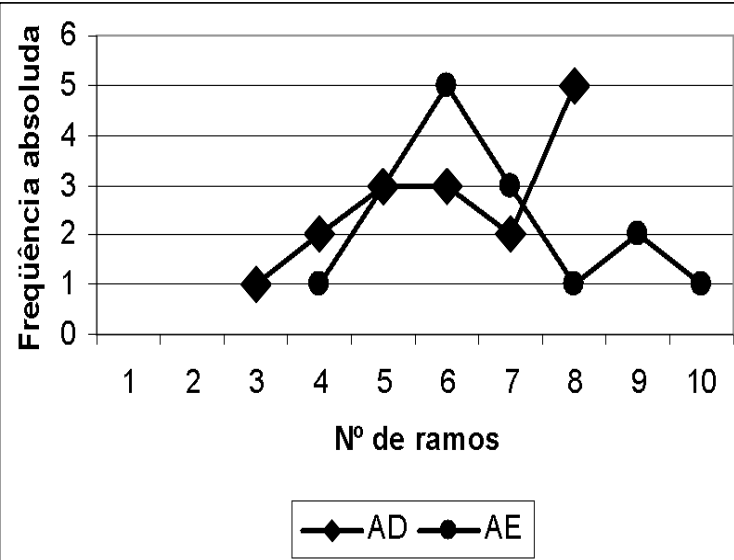

Figura 2 - Freqüência absoluta do número de ramos linguais primários do nervo lingual por antímero esquerdo (AE) e direito (AD) em língua de suínos (Sus scrofa doméstica - Linnaeus, 1758), Goiânia - 2007. Fonte: Dados de Pesquisa UFG/UnB.
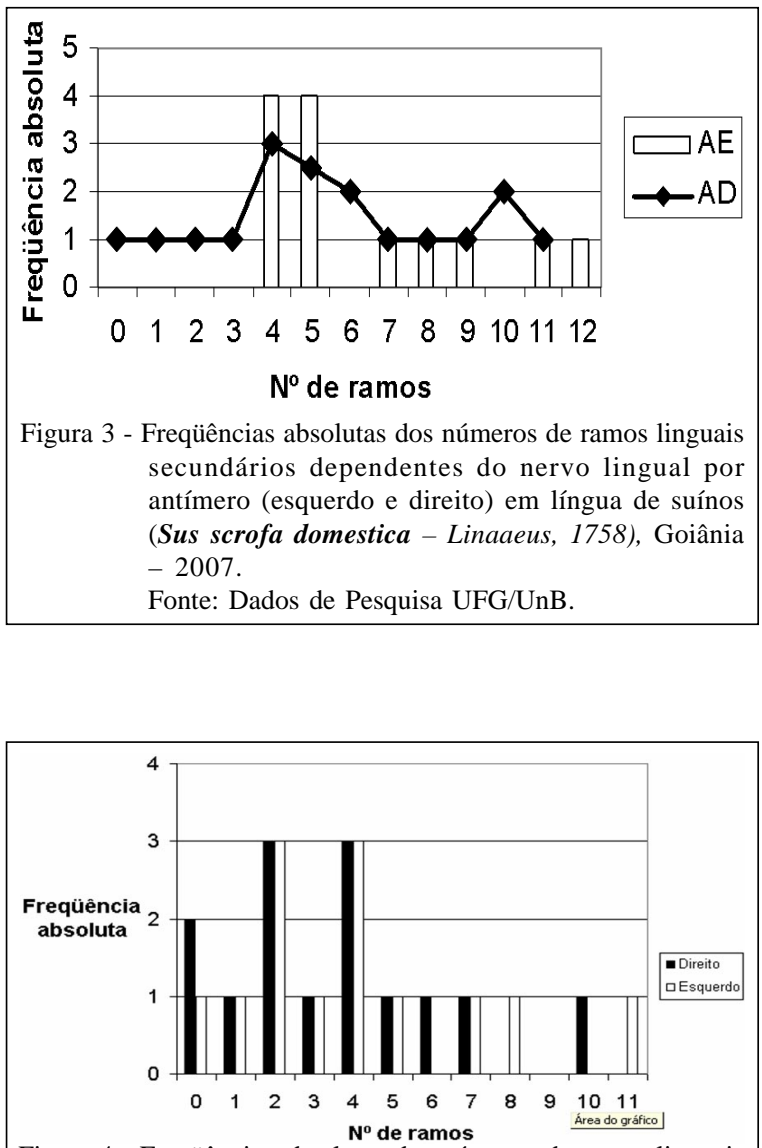

Figura 4 - Freqüências absolutas dos números de ramos linguais terciários dependentes do nervo lingual por antímero (esquerdo e direito) em línguas de suínos (Sus scrofa doméstica, Linnaeus, 1758), Goiânia - 2007. Fonte: Dados de Pesquisa UFG/UnB.. 


\section{REFERÊNCIAS}

BITTENCOURT, J.C. et al. The anatomy of the terminal branches of the lingual nerve (lingual branches) in adult brasilians. Revista Brasileira de Ciências Morfológicas, v.4, n.2, p.115-121, 1987.

BLANDIN, F.P. Noveaux elements d'anatomie descriptive. Paris: J.B. Bailliere, 1938. V.2, p.615-617.

BRUNI, A.C.; ZIMMERL, V. Anatomia degli animali domestici. 2.ed. Milano: Francesco Villardi, 1951. 735p..

CASTELLI, W.A.; HUELKE, D.F. The arterial system of the head and neek of the Rhesus monkey with empliasis on the external carotid system. American Journal of Anatomy, v.116, n.1, p.149-170, 1964.

DYCE, K.M. et al.. Tratado de anatomia veterinária. Rio de Janeiro: Guanabara Koogan, 1990. 813 p.

EVANS, H.E.; CHRISTENSEN, G.C. Miller's anatomy of the dog. 2.ed. Philadelphia: Saunders, 1979. 1113 p..

FAVAROLO, S. Manuale di anatomia topografica. 2.ed. Napoli: Stamperia Gia Del Fibreno, 1979. 576 p.

GETTY, R. Anatomia de los animales domésticos. 5.ed. Barcelona: Salvat, 1981. Cap.1, p.508-556.

INTERNACIONAL COMMITEE ON VETERINARY GROSS ANATOMICAL NOMENCLATURE. Nomina anatômica veterinaria. 4.ed. Zurich, 1994. p.43-44.

MADEIRA, M.C. Anatomia da face: bases anatomofuncionais para a prática odontológica. 5.ed. rev. e ampl. São Paulo: Sarvier, 2004. p.133-134.

MIKHAIL, Y. et al., Observations on the structure of the subepitelial nerve plexus in the tongue. Acta Anatomica., v.107, p.311-317, 1980.
OKANO, S. Inervation especially sensory innervation of dog tonge. Tohoku Journal Exerpta. Medica, v.57, p.169-79, 1953.

SCALZI, H.A. The cytoarquiteture of gustatory receptors from the rabit foliate papillae. Zeit, Zellforsch, v.80, p.413-435, 1967.

SCHALLER, O. Nomenclatura Anatômica Veterinária Ilustrada. São Paulo: Manole, 1999. 614p.

SCHAWARZE, E.; SCHRÖDER, L. Compêndio de Anatomia Veterinária. Zaragoza: Acriba, 1970.

SEMPRINI, M. et al. . Observações sobre as terminações nervosas da mucosa lingual em cobaias. Estudo ao microscópio ótico. Ciência e Cultura, v.37, p.1940-1943, 1985.

SONNTAG, C.F. The comparative anatomy of the tongues of the mammalia, XII. Summary, classification and phylageny. Proceeding Zoological Society London.p.701-762, 1925.

SONNTAG, C.F. The comparative anatomy of the tongue of the mammalia. VII. Cetacea, Sirienia and ungulata. Proceeding Zoological Society London. p.639-657, 1922.

SONNTAG, C.F. The comparative anatomy of the tongue of the mammalia. I. general description of the tongue. Proceeding Zoological Society London. p.115-129, 1920.

VIG, S.; KANAGASUNTHERAM, R. Innervation of oral tissues in some primates. Folia Primatologica, v.11, p.289-299, 1969.

WATANABE, I.; KONIG JR, B. Neurohistological observations on the tongue of the tufed capuchin. Cebus apella, Linnaeus, 1758. Revista Brasileira de Pesquisas Médicas e Biológicas, v.10, p.121-127, 1977. 